\title{
EQUIVARIANT GEOMETRY AND KERVAIRE SPHERES
}

\author{
ALLEN BACK AND WU - YI HSIANG
}

\begin{abstract}
The intrinsic geometry of metrics on the Kervaire sphere which are invariant under a large transformation group (cohomogeneity one) is studied. Invariant theory is used to describe the behavior of the metrics near the singular orbits. Nice expressions for the Ricci and sectional curvatures are obtained. The nonexistence of invariant metrics of positive sectional curvature is proven, and Cheeger's construction of metrics of positive Ricci curvature is discussed.
\end{abstract}

I. Introduction. In $[\mathbf{B d C H}]$, a general framework for equivariant differential geometry was sketched. In this paper, we apply that framework and study all invariant metrics on a class of manifolds including the Kervaire sphere $\Sigma^{4 n+1}$ with an almost transitive (cohomogeneity one) $S^{1} \times O(2 n+1)$ action.

$\S \S I I$ and III review the general approach to studying $G$-invariant metrics on a manifold $M$ as suggested in [BdCH]. §IV makes this explicit in the case of the Kervaire sphere; particularly interesting is the somewhat delicate way that the invariant theory of the slice representations at nonprincipal orbits dictate the possibilities for smooth invariant metrics.

In $\S \mathrm{V}$, the Ricci and sectional curvature of arbitrary left invariant metrics on $S^{1} \times S O(n) / S O(n-2)$ (principal orbits of the action) are calculated. §VI uses these results to obtain curvature computations for arbitrary invariant metrics on Kervaire spheres. The answers are remarkably simple.

We apply these considerations to the question of whether or not Kervaire spheres possess equivariant metrics of positive curvature. Away from one orbit, the Kervaire sphere is equivariantly diffeomorphic to the standard sphere with an orthogonal action, yet we find that positive sectional curvature is impossible for an invariant metric (VI.3). Cheeger [C] has shown the existence of invariant metrics with positive Ricci curvature, and in §VII we relate his construction to our framework.

We also make use of the curvature formulas for $S^{1} \times S O(n) / S O(n-2)$ to show the nonexistence of invariant Einstein metrics on this homogeneous space (V.6) as well as the uniqueness of Sagle's Einstein metric on $S O(n) / S O(n-2)$ (V.7).

In all sign conventions, we follow Kobayashi-Nomizu [KN].

Received by the editors June 2, 1986.

1980 Mathematics Subject Classification (1985 Revision). Primary 53C20, 58B20; Secondary 53C30, $53 \mathrm{C} 25,57 \mathrm{~S} 25$.

The work of both authors supported by NSF grants. 
II. Geometry of orbit structures. First we recall the geometry of a complete $G$-Riemannian manifold $M$ as described in $[\mathbf{B d C H}$. Here $M$ is a smooth Riemannian manifold on which a Lie group $G$ acts through isometries. Let $H$ be a fixed principal isotropy subgroup. The union $M_{0}$ of the principal orbits (those with isotropy group conjugate to $H$ ) forms the regular part of $M$ and has the structure of a $G / H$ fiber bundle over $M_{0} / G$. The complement $M_{s}=M-M_{0}$ is the singular set. The fixed point set $\bar{M}_{0}$ of $H$ acting on $M_{0}$ is the total space of a $\bar{G}=N(H) / H$ principal bundle. The $\bar{G}$ action extends to the closure $\bar{M}$ of $\bar{M}_{0}$, and as $\bar{M}$ cuts every $G$-orbit, $G$-equivariant information about $M$ may generally be reduced to $\bar{G}$ equivariant data at points of $\bar{M} . \bar{M}_{0}$ is the regular part of $\bar{M}$.

In the diagram

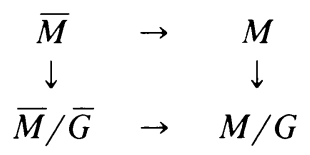

the vertical maps are Riemannian submersions along the regular sets. The submersed metric measures the distance between orbits. Off the singular set, $\bar{M} / \bar{G} \rightarrow M / G$ is an isometry. The orthogonal complement to the orbits in $\bar{M}_{0} \rightarrow \bar{M}_{0} / \bar{G}$ determines a $\bar{G}$-connection in the principal bundle whose curvature is essentially the fundamental tensor of the submersion (as in [ON]) $M_{0} \rightarrow M_{0} / G$ evaluated at points of $\bar{M}$.

A function $f: M / G \rightarrow R$ is defined to be smooth if the composition $\bar{f}: M \rightarrow$ $M / G \rightarrow R$ is smooth. In understanding this smooth structure, the following basic theorem of Schwarz [Sc] is fundamental since it points out the intimate relationship between smooth functions near the singular set and the invariant theory of the slice representations.

THEOREM II.1. For any given point $x \in M$ with isotropy group $G_{x}$, let $S_{x}$ be the linear $G_{x}$-space of all normal vectors to the orbit $\xi=G(x)$ at $x$ (i.e. the slice). Let $\left\{\sigma_{1}, \sigma_{2}, \cdots, \sigma_{l}\right\}$ form a Hilbert basis of the ring $R\left[S_{x}\right]^{G_{x}}$ of $G_{x}$-invariant polynomials. Then the $\left\{\sigma_{i}\right\}$ form a local coordinate system for a suitable neighborhood of $\xi$ in $M / G$; i.e. all $C^{\infty}$-invariant functions near $\xi$ are $C^{\infty}$ functions of the $\sigma_{i}$.

Let $g$ and $h$ be the Lie algebras of $G$ and $H$, respectively. Choose a fixed Ad ${ }_{r}$-invariant positive definite quadratic form on $g$ and let $\not h$ be the orthogonal complement of $h$ in $g$ with respect to this form. Then at each point of $\bar{M}_{0}$, the orbit of $G$ is naturally identified with $G / H$ and the metric induced from $M$ on this orbit is given by an $\mathrm{Ad}_{H}$-invariant inner product on $\not h$. Furthermore, the Schur lemma tells us that the number of distinct left invariant metrics on $G / H$ is determined by the decomposition $\sum_{\alpha=1}^{r} n_{\alpha} \rho_{\alpha}$ of the linear isotropy representation into distinct irreducible representations $\rho_{\alpha}$ of $H$. (The $n_{\alpha}$ are multiplicities.) More precisely the representation spaces corresponding to nonisomorphic irreducibles are orthogonal, and the bilinear form restricted to pairs of vectors in isomorphic irreducible representation spaces must be (after identification) a multiple of any choice 
(e.g. the trace form) of $H$-invariant quadratic form. So an arbitrary $\mathrm{Ad}_{H^{r}}$ invariant quadratic form on $\not h$ is given by an element of $\oplus_{\alpha=1}^{r} \mathrm{GL}^{s}\left(n_{\alpha}, R\right) \subseteq$ $\mathrm{GL}^{s}(m, R)$, where $\mathrm{GL}^{s}(k, R)$ denotes the nonsingular symmetric $k \times k$ real matrices and $m=\sum_{\alpha=1}^{r} n_{\alpha} \operatorname{dim}\left(\rho_{\alpha}\right)$.

So the tangential components of the metric are determined by a function $h$ : $\bar{M} \rightarrow \mathrm{GL}^{s}(m)$. It is important to realize that this function is $\bar{G}$-covariant. Explicitly, let $\left\{V_{j}: 1 \leqslant j \leqslant m\right\}$ be a fixed and chosen basis for $\not, B(n)$ the matrix of $\operatorname{Ad}(n)$ acting on $\not h$ with respect to this basis, and $h_{i j}(x)=\left\langle V_{i}^{*}(x), V_{j}^{*}(x)\right\rangle$ where $V_{i}^{*}(x)$ is the associated Killing field at $x$. (We drop the $*$ when no confusion will result.) Then, because $V_{i}^{*}(n x)=L_{n}\left(\left(\operatorname{Ad}\left(n^{-1}\right) V_{i}\right)^{*}(x)\right), h$ satisfies the covariance property.

Proposition II.2. $h(n x)=B\left(n^{-1}\right)^{t} h(x) B\left(n^{-1}\right)$.

The function $h$ also determines the second fundamental form of each principal orbit (see Proposition III.1).

The above discussion naturally leads to the following uniqueness theorem showing that the $G$-isometry type of a $G$-Riemannian manifold is determined by the three basic invariants consisting of: (a) submersed orbital distance metric on $M_{0} / G$; (b) a $\bar{G}$-connection on the principal bundle $\bar{M}_{0} \rightarrow M_{0} / G\left(=\bar{M}_{0} / \bar{G}\right)$; (c) a matrix-valued covariant function $h_{i j}(x)$ on $\bar{M}_{0}$.

THEOREM II.3. Suppose that $M$ and $M^{\prime}$ are two complete Riemannian G-manifolds with the same principal orbit type $G / H$. Assume there exists a $\bar{G}$-equivariant map $\bar{i}$ : $\bar{M}_{0} \rightarrow \bar{M}_{0}^{\prime}$ satisfying

(a) the induced map $\tilde{i}: M_{0} / G \rightarrow M_{0}^{\prime} / G$ is an isometry.

(b) $i^{*}$ preserves the $\bar{G}$-connections.

(c) $\bar{i}^{*}$ preserves the matrix-valued functions $h_{i j}$ and $h_{i j}^{\prime}$.

Then there is an equivariant isometry $i: M \rightarrow M^{\prime}$ whose induced map on orbit spaces is exactly $\tilde{i}$.

Equally important, the three basic invariants may be used to construct examples of $G$-Riemannian manifolds. The basic tool is the slice theorem describing a tubular neighborhood of any orbit $G(x)$. The slice $S$ at $x$ is the intersection of an open ball about 0 in $T_{x} M$ with the set of normal vectors to the orbit $G(x)$. Then using the exponential map, a tubular neighborhood of $G(x)$ is identified up to equivariant diffeomorphism with the bundle $G \times{ }_{K} S \rightarrow G / K$ where $K$ is the isotropy group at $x$. The action of $K$ on $S$ is orthogonal and is called the slice representation at $x$. The projection $G \times{ }_{K} S \rightarrow K / S$ is just a local version of the orbit map, and principal orbits are exactly the places where the slice representation is trivial.

If $X$ is a smooth $K$-manifold and $Y$ is a smooth $G$-manifold with $K$ a subgroup of $G$, it is immediate that any smooth $K$-equivariant map $\theta: X \rightarrow Y$ has a smooth extension to a $G$-map $\tilde{\theta}: G \times{ }_{K} X \rightarrow Y$. Using this, it is easy to see

Proposition II.4. Let $S$ be the slice at a point $x$ with isotropy group $K$. Then any $K$-invariant smooth inner product defined on $T_{y} M$ for all $y \in S$ has a (unique) smooth extension to a $G$-invariant Riemannian metric on a neighborhood of $G(x)$. 
So checking the smoothness of the metric determined by the basic invariants need only be done at points of a slice. As a consequence, in the special case when $M$ has only one orbit type ( $M_{s}=\varnothing$ and the slice representation is trivial), we get the following simple existence theorem.

Proposition II.5. Suppose $M$ is a given G-manifold with all orbits of the same type $G / H$. Then an arbitrary set of geometric invariants of type (a), (b), and (c) can be realized by a suitable G-Riemannian structure on $M$. In fact, any smooth choice of $\mathrm{Ad}_{H}$-invariant inner product on $\not \mathrm{c}$ at points of $S$ has a smooth extension to an $N(H)$-covariant function $h$ of type (c) defined on the open set $\bar{G}(S)$ of $\bar{M}$.

The above proposition always applies to the regular part $M_{0}$ of any $G$-Riemannian manifold $M$. At points of $M_{s}$, the actual application of II.4 depends on the understanding of smooth $K$-equivariant maps between linear representations. (The failure of the equivariant Luna-Richardson theorem prevents a complete reduction of the smoothness condition to $\bar{G}$-slices in $\bar{M}$.) The smooth $\left(C^{\infty}\right) K$-equivariant functions between linear representations form a module over the smooth real-valued invariant functions on the domain. A module basis is given by a module basis for the equivariant polynomials as a module over the invariant polynomials, and the smooth invariant functions are given by Schwarz's theorem.

We will need the cases of circle and cyclic actions, so we state the following proposition readily proven by averaging:

Proposition II.6. (1) Let $S^{1}$ act on $C^{n}=\left\{\left(z_{1}, \ldots, z_{n}\right): z_{i} \in C\right\}$ by $\lambda\left(z_{1}, \ldots, z_{n}\right)$ $=\left(\lambda^{m_{1}} z_{1}, \ldots, \lambda^{m_{n}} z_{n}\right)$ and on $C$ by $\lambda(z)=\lambda^{m_{0}} z$. Then the equivariant polynomial maps from $C^{n}$ to $C$ have as module basis (over $C$ ) the monomials $z_{1}^{\alpha_{1}} \cdots z_{n}^{\alpha_{n}} \bar{z}_{1}^{\beta_{1}} \cdots \bar{z}_{n}^{\beta_{n}}$ with $\sum_{i=1}^{n} m_{i}\left(\alpha_{i}-\beta_{i}\right)=m_{0}$.

(2) If we restrict the above actions to the cyclic subgroup $Z_{k}$, then the equivariant polynomials are additively generated by the monomials with $\sum_{i=1}^{n} m_{i}\left(\alpha_{i}-\beta_{i}\right)-m_{0}$ divisible by $k$.

III. Curvatures in orbit map submersions. Here we recall the basic curvature formulas of $[\mathbf{B d C H}]$ which specialize those of $[\mathbf{O N}]$ to the case of invariant metrics. Firstly, the second fundamental form of the fiber is determined by the covariant function $h$.

Proposition III.1. If $X$ is horizontal and $V, W$ are vertical (Killing) vector fields, then

$$
\left\langle T_{V} W, X\right\rangle=-\frac{1}{2} L_{X}\langle,\rangle(V, W) .
$$

Thus $\left\langle T_{V}, V_{j}, X\right\rangle=-\frac{1}{2} X h_{i j}$ in the notation of $\S I$ II.

The fundamental method of describing invariant connections on a homogeneous space $G / H$ is to use the following relation $[\mathbf{K N}]$, due to Wang:

$$
\nabla_{V_{1}^{*}} V_{2}^{*}=\frac{1}{2}\left[V_{1}^{*}, V_{2}^{*}\right]+U\left(V_{1}, V_{2}\right)^{*},
$$

where $V_{i} \in \not$ and $2\left\langle U\left(V_{1}, V_{2}\right), V_{3}\right\rangle=\left\langle\left[V_{3}, V_{1}\right]_{\not}, V_{2}\right\rangle+\left\langle V_{1},\left[V_{3}, V_{2}\right]_{\mu}\right\rangle$. (Here $V_{i} \in \not h$, $V_{i}^{*}$ is the associated Killing field, and $\left[V_{i}, V_{j}\right]^{*}=-\left[V_{i}^{*}, V_{j}^{*}\right]$.) 
In the almost homogeneous case $\left(\operatorname{dim} M_{0} / G=1\right)$, the fundamental tensor $A$ of the submersion, being a curvature, is zero. Let $C: \bar{M}_{0} \rightarrow R$ be the orbital volume element function; $C^{2}=\operatorname{det} h$. Then O'Neill's curvature formulae become [BdCH]

Proposition III.2. If $M$ is almost homogeneous, the $V_{i}$ vertical Killing, and $\hat{L} a$ unit horizontal, then

$$
\begin{aligned}
\left\langle R\left(V_{1}, V_{2}\right) V_{3}, V_{4}\right\rangle= & \left\langle\hat{R}\left(V_{1}, V_{2}\right) V_{3}, V_{4}\right\rangle \\
& +\frac{1}{4}\left[\hat{L}\left\langle V_{1}, V_{3}\right\rangle \hat{L}\left\langle V_{2}, V_{4}\right\rangle-\hat{L}\left\langle V_{1}, V_{4}\right\rangle \hat{L}\left\langle V_{2}, V_{3}\right\rangle\right], \\
\left\langle R\left(V_{1}, \hat{L}\right) \hat{L}, V_{2}\right\rangle=-\frac{\hat{L} \hat{L}\left\langle V_{1}, V_{2}\right\rangle}{2}+\left\langle T_{V_{1}} \hat{L}, T_{V_{2}} \hat{L}\right\rangle, & \\
\left\langle R\left(V_{1}, V_{2}\right) V_{3}, \hat{L}\right\rangle= & \frac{1}{2}\left[\left\langle\left[\hat{L}, U\left(V_{2}, V_{3}\right)\right], V_{1}\right\rangle-\left\langle\left[\hat{L}, U\left(V_{1}, V_{3}\right)\right], V_{2}\right\rangle\right], \\
\operatorname{Ric}\left(V_{1}, V_{2}\right)= & \widehat{\operatorname{Ric}}\left(V_{1}, V_{2}\right)-\frac{\hat{L} \hat{L}}{2}\left\langle V_{1}, V_{2}\right\rangle \\
& +\frac{\hat{L} \log C \hat{L}\left\langle V_{1}, V_{2}\right\rangle}{2}+2\left\langle T_{V_{1}} \hat{L}, T_{V_{2}} \hat{L}\right\rangle .
\end{aligned}
$$

Here $\widehat{\text { Ric }}$ and $\hat{R}$ denote the curvature functions of the orbit. The orbital volume element function $C$ enters in the above Ricci tensor formula because of the well-known $\operatorname{Tr}\left(h^{-1} \hat{L} h\right)=\hat{L}(\log \operatorname{det} h)$ for any matrix-valued function $h$. In using the above formulae, note that

$$
\left\langle T_{V_{i}} \hat{L}, T_{V_{j}} \hat{L}\right\rangle=\frac{1}{4} \sum_{k, p} h^{k p} \hat{L} h_{i k} \hat{L} h_{j p}
$$

where $h^{k p}$ is the inverse matrix of $h_{i j}$. Also, for two-by-two matrices $h$,

$$
(\hat{L} h) h^{-1}(\hat{L} h)=(\hat{L} h)(\hat{L} \log \operatorname{det}(h))-\frac{h \operatorname{det}(\hat{L} h)}{\operatorname{det}(h)} .
$$

IV. The Kervaire example. We now apply the above discussion to a specific example which includes the Kervaire sphere $\Sigma_{l}^{2 n-1}$ ( $n$ odd) defined as a Brieskorn variety in $C^{n+1}$. Explicitly, if $C^{n+1}=C^{1} \times C^{n}$ has coordinates $\left(z_{0}, Z\right)$ where $Z=\left(z_{1}, \ldots, z_{n}\right)$, then $\Sigma_{l}$ is the set of zeros of $z_{0}^{l}+\sum_{i=1}^{n}\left(z_{i}\right)^{2}=0$ and $\sum_{i=0}^{n}\left|z_{i}\right|^{2}=1$. If we let $X=\operatorname{Re}(Z)$ and $Y=\operatorname{Im}(Z)$, these equations take the form

$$
\begin{aligned}
& \operatorname{Re}\left(z_{0}^{l}\right)+X \cdot X-Y \cdot Y=0, \\
& \operatorname{Im}\left(z_{0}^{l}\right)+2 X \cdot Y=0, \\
& \left|z_{0}\right|^{2}+X \cdot X+Y \cdot Y=1,
\end{aligned}
$$

where denotes the ordinary Euclidean inner product. $C^{n+1}$ carries a $G=S^{1} \times O(n)$ action defined by

$$
\left(e^{i \theta}, g\right)\left(z_{0}, X, Y\right)=\left(e^{2 i \theta} z_{0}, \cos l \theta g X-\sin l \theta g Y, \sin l \theta g X+\cos l \theta g Y\right)
$$

which is easily seen to induce an action on $\Sigma$. 
The $O(n)$-invariant polynomials on $C^{n+1}$ are generated by $x_{0}, y_{0}, \alpha=$ $\frac{1}{2}(X \cdot X+Y \cdot Y), u=\frac{1}{2}(X \cdot X-Y \cdot Y)$, and $v=X \cdot Y$. By studying the remaining $S^{1}$ action on these and setting $w=u+i v$ (note $w=\frac{1}{2} \sum_{i=1}^{n} z_{i}^{2}$ ), we see that a Hilbert basis for the $G$-invariant polynomials is given by

$$
\begin{gathered}
\alpha=\frac{1}{2}(X \cdot X+Y \cdot Y), \quad \beta=\left|z_{0}\right|^{2}, \quad \gamma=u^{2}+v^{2}=|w|^{2}, \\
\sigma=\operatorname{Re} z_{0}^{l} \bar{w}, \quad \tau=\operatorname{Im} z_{0}^{l} \bar{w} .
\end{gathered}
$$

The above five basic invariant functions imbed the orbit space $C^{n+1} / G$ into $R^{5}$ with image the open cone over a topological 3-disc.

From now on, let us assume $l$ is odd. Every orbit on $\Sigma_{l}$ contains points with $\operatorname{Im}\left(z_{0}\right)=0$, so it is easy to see that the orbit space $\Sigma_{l} / G$ is a curve in $C^{n+1} / G$. Reparameterizing, the projection map $\Sigma_{l} \rightarrow\left[0, \rho_{0}^{2}\right]$ is induced by $\left(z_{0}, Z\right) \mapsto\left|z_{0}\right|^{2}$ where $\rho_{0}$ is the positive real root of $\rho_{0}^{2}+\rho_{0}^{l}=1$. The principal isotropy group is $H=Z_{2} \times O(n-2)$. At $z_{0}=0, X$ and $Y$ are orthogonal of the same length and we have the isotropy group $H_{0}=S^{1} \times O(n-2) \subset S^{1} \times O(2) \times O(n-2)$, where the $S^{1}$ sits in $S^{1} \times O(2)$ by $e^{i \theta} \mapsto\left(e^{i \theta}, R_{l \theta}\right)$, where $R_{l \theta}$ is the matrix of rotation counterclockwise by $l \theta$. When $z_{0}=\rho_{0}, \operatorname{span}(X, Y)$ becomes one-dimensional and the isotropy group $H_{1}=Z_{2} \times O(n-1) \subset S^{1} \times O(1) \times O(n-1)$ with $Z_{2} \subset S^{1} \times$ $O(1)$ by the diagonal.

A natural choice of complement $\not \underline{h}$ of $\underline{h}$ in $\underline{g}$ is the span of vectors

$$
\begin{gathered}
P=O_{2}^{1}, \quad Q=(\partial / \partial \theta)^{*}, \\
E_{i}=O_{1}^{i}, \quad F_{i}=O_{2}^{i}, \quad 3 \leqslant i \leqslant n,
\end{gathered}
$$

where $O_{i}^{j}$ is the skew-symmetric $n \times n$ matrix with +1 in row $i$, column $j$, and zeros elsewhere above the diagonal, and $\partial / \partial \theta$ is the tangent to the $S^{1}$ factor in $S^{1} \times O(n)$. Thus the principal orbit metric is is determined by the two symmetric matrix-valued covariant functions $a$ and $b$ defined by

$$
\begin{aligned}
& a=\left(\begin{array}{ll}
a_{11} & a_{12} \\
a_{21} & a_{22}
\end{array}\right)=\left(\begin{array}{ll}
\left\langle E_{i}, E_{i}\right\rangle & \left\langle E_{i}, F_{i}\right\rangle \\
\left\langle E_{i}, F_{i}\right\rangle & \left\langle F_{i}, F_{i}\right\rangle
\end{array}\right), \\
& b=\left(\begin{array}{ll}
b_{11} & b_{12} \\
b_{21} & b_{22}
\end{array}\right)=\left(\begin{array}{cc}
\langle P, P\rangle & \langle P, Q\rangle \\
\langle P, Q\rangle & \langle Q, Q\rangle
\end{array}\right) .
\end{aligned}
$$

We shall use the notations $A=\operatorname{det}(a)$ and $B=\operatorname{det}(b)$.

The manifold $\bar{M}=F\left(H, \Sigma_{l}\right)$ is generally a Lens space $\Sigma_{l} \cap\left(C^{1} \times C^{2}\right)$ and this is the natural domain of the functions $a_{i j}$ and $b_{i j}$. The group $\bar{G}=N(H) / H$ is $S^{1} \times O(2)$.

The slice representations for $\bar{G}$ and $G$ are also easy to calculate. At $z_{0}=0$, the slices for both $\bar{G}$ and $G$ are two dimensional and the isotropy $S^{1}$ winds around twice. At $z_{0}=\rho_{0}$, the nontrivial part of the slice for the $G$ action is given by the standard action of $O(n-1)$ on $R^{n-1}$. The nontrivial part of the $\bar{G}$-slice is given by $Z_{2}$ acting as a one-dimensional reflection.

The slice representations tell us exactly which functions $a$ and $b$ are realizable for equivariant metrics on $\Sigma_{l}$. At $z_{0}=0$, the slice is a two-dimensional vector space with radial lines corresponding to horizontal geodesics. Let $z$ be the natural complex 
coordinate in the slice so that $x e_{1}+y e_{2}$ is a unit horizontal vector $\left(e_{1}=\partial / \partial x, x=\right.$ $\operatorname{Re}(z) ; e_{2}, y$ similarly). The covariance condition (Proposition II.3) requires that the $b_{i j}$ and $a_{11}+a_{22}$ be invariant functions on the slice while $a_{11}-a_{22}+2 i a_{12}$ is covariant with respect to rotation $l$ times faster in the range than in the domain. Thus near $z=0$, there must be a smooth function $\tau: R \rightarrow C$ so that $a_{11}-a_{22}+$ $2 i a_{12}=z^{l} \tau\left(|z|^{2}\right)$ and the invariant functions must be smooth functions of $|z|^{2}$.

Since the orbit space is one dimensional, the functions $a$ and $b$ are determined by their restrictions $\tilde{a}$ and $\tilde{b}$ to a single horizontal geodesic (radial ray) emanating from the orbit at $z_{0}=0$. Letting $r$ denote the unit speed parameter along this geodesic $\left(r^{2}=|z|^{2}\right)$, the above conditions become

$$
\tilde{a}_{11}-\tilde{a}_{22}=r^{\prime} \tau_{1}\left(r^{2}\right), \quad \tilde{a}_{12}=r^{\prime} \tau_{2}\left(r^{2}\right) / 2, \quad \tilde{a}_{11}+\tilde{a}_{22}=\tau_{3}\left(r^{2}\right),
$$

where the $\tau_{i}$ are $C^{\infty}$ functions $\left(\tau_{1}=\operatorname{Re}(\tau), \tau_{2}=\operatorname{Im}(\tau)\right)$. The $\tilde{b}_{i j}$ are all $C^{\infty}$ functions of $r^{2}$.

However, because radial vector fields are singular at the origin, smoothness of the functions $a$ and $b$ does not guarantee a smooth metric on $S$. We must translate the equivariance conditions above into conditions on the inner products of a spanning set of vector fields. An easy calculation shows that

$$
\begin{gathered}
\left\langle P^{*}, e_{1}\right\rangle=-\frac{y}{2 r^{2}}\left(b_{12}+l b_{11}\right), \quad\left\langle Q^{*}, e_{1}\right\rangle=\frac{-y}{2 r^{2}}\left(b_{22}+l b_{12}\right), \\
\left\langle e_{1}, e_{1}\right\rangle=\frac{y^{2}}{4 r^{4}}\left(b_{22}+2 l b_{12}+l^{2} b_{11}\right)+\frac{x^{2}}{r^{2}} .
\end{gathered}
$$

These show us that

$$
\tilde{b}(r)=\left(\begin{array}{ll}
\tilde{b}_{11} & \tilde{b}_{12} \\
\tilde{b}_{12} & \tilde{b}_{22}
\end{array}\right)=b_{0}\left(\begin{array}{cc}
1 & -l \\
-l & l^{2}
\end{array}\right)+r^{2}\left(\begin{array}{ll}
\hat{b}_{11} & \hat{b}_{12} \\
\hat{b}_{12} & \hat{b}_{22}
\end{array}\right)+r^{4}\left(\begin{array}{ll}
\bar{b}_{11} & \bar{b}_{12} \\
\bar{b}_{12} & \bar{b}_{22}
\end{array}\right),
$$

where $b_{0}, \hat{b}_{i j} \in R$, the $\bar{b}_{i j}$ are smooth functions of $r^{2}$, and $l^{2} \hat{b}_{11}+2 l \hat{b}_{12}+\hat{b}_{22}=4$. It is easy to check that these conditions are sufficient for smoothness and together with positive definiteness are the only conditions on the functions $a$ and $b$.

Suppose the distance between the singular orbits is $L$. Set $\bar{r}=L-r$, where $r$ as above is the distance to the orbit at $z_{0}=0$. Let us analyze the behavior of the functions $a$ and $b$ near a point with isotropy group $H_{1}=Z_{2} \times O(n-1) \subseteq S^{1} \times$ $O(n)$ as described earlier. Choose an orthonormal basis $e_{1}, \ldots, e_{n-1}$ for the slice $S=R^{n-1}$ with associated coordinates $x_{1}, \ldots, x_{n-1}$. Then $\sum_{i=1}^{n-1}\left(x_{i}\right)^{2}=\bar{r}^{2}$. We again use the notation $\tilde{a}$ and $\tilde{b}$ for the restrictions of $a$ and $b$, respectively, to a radial geodesic lying in the $\bar{G}_{1}=N\left(H_{1}\right) / H_{1}$ slice.

The $\bar{G}$ slice is one dimensional and it is easy to see that covariance demands that $\tilde{a}_{12}$ and $\tilde{b}_{12}$ be odd functions of $\bar{r}$ while $a_{i i}$ and $b_{i i}$ are even functions. But it turns out that this is an example where the equivariant Luna-Richardson theorem does not hold. 
By choosing an explicit rotation from $\bar{r} e_{1}$ to an arbitrary point $x=\left(x_{1}, \ldots, x_{n-1}\right)$ $\in S$, we can verify that at $x$,

$$
\begin{gathered}
\left\langle E_{i}, P\right\rangle=\frac{x_{1} x_{i}}{\bar{r}^{2}}\left(\tilde{b}_{11}-\tilde{a}_{11}\right), \quad\left\langle F_{i}, P\right\rangle=\frac{x_{i}}{\bar{r}} \tilde{a}_{12}, \\
\left\langle e_{i}, e_{j}\right\rangle=\frac{x_{i} x_{j}}{\bar{r}^{2}}\left(1-\frac{\tilde{a}_{22}}{\bar{r}^{2}}\right)+\delta_{i j} \frac{\tilde{a}_{22}}{\bar{r}^{2}}, \quad\left\langle e_{i}, P\right\rangle=x_{1} x_{i} \frac{\tilde{a}_{12}}{\bar{r}^{3}} .
\end{gathered}
$$

Smoothness of the metric and the first equation require that $\tilde{b}_{11}=\tilde{a}_{11}$ at $r=L$. This is just an infinitesimal version of the requirement that we have a $G$-invariant metric on $G / H_{1} \sim S^{1} \times S^{n-1}$. The second equation forces $\tilde{a}_{12}$ to vanish to first order, but the fourth requries vanishing to third order. Thus $\tilde{a}_{11}=\sigma_{1}\left(\bar{r}^{2}\right), \tilde{a}_{12}=$ $\bar{r}^{3} \sigma_{2}\left(\bar{r}^{2}\right)$, and $\tilde{a}_{22}=\bar{r}^{2}+\bar{r}^{4} \sigma_{3}\left(\bar{r}^{2}\right)$ (where the $\sigma_{i}$ are $C^{\infty}$ ). One can verify that these conditions together with the previously mentioned even/odd conditions on the $\tilde{b}_{i j}$ characterize smooth metrics.

We could translate these conditions to other points of the singular orbit at $r=L$, but for geometric reasons this is not necessary here. The horizontal geodesic in $\bar{M}$ emanating from a point with isotropy group exactly the above $H_{1}$ (as opposed to some conjugate of $H_{1}$ ) eventually becomes a radial line in a slice with isotropy group $S^{1} \times O(n-2)$ at $r=0$. Because different points in the singular orbit $G / H_{1}$ with the same isotropy subgroup $H_{1}$ differ by the action of an element of the center of $G$, the functions $\tilde{a}$ and $\tilde{b}$ do not depend on which point with isotropy group $H_{1}$ we consider. Because all slices along the other singular orbit $G / H_{0}$ are geometrically equivalent (i.e., elements of $N\left(H_{0}\right)$ - and $H_{0}$-equivariant linear maps of $S$ induce enough equivariant diffeomorphisms to take a radial line in one slice to any other radial line in any other slice along this orbit) we see that the equivariant isometry class is independent of anything other than the functions $\tilde{a}$ and $\tilde{b}$. Thus we have

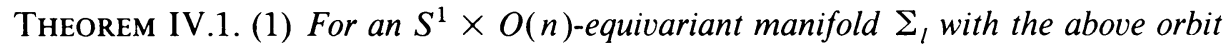
structure, the equivariant isometry class is uniquely determined by the distance $L$ and the functions $\tilde{a}$ and $\tilde{b}$ along a horizontal geodesic between two singular points with isotropy groups $H_{0}$ and $H_{1}$.

(2) Any set of functions $\tilde{a}$ and $\tilde{b}$ on $[0, L]$ with the above discussed vanishing properties at $r=0$ and $r=L$ (and positive definiteness conditions) give a smooth equivariant metric on $\Sigma$.

It is interesting to note that the standard constant curvature sphere in the $S^{1} \times O(n)$ representation space $R^{2} \otimes R^{n}$ has the orbit structure of $\Sigma_{l}$ for $l=1$. Thus the manifolds $\Sigma$, are all equivariantly diffeomorphic to the sphere $S^{2 n-1}$ away from one orbit. It is the imbedding of the $S^{1}$ factor into $S^{1} \times O(2)$ at this orbit which determines the topological type of $\Sigma_{l}$. When $l= \pm 3(\bmod 8), \Sigma_{l}$ is a Kervaire sphere, $l= \pm 1(\bmod 8)$ makes $\Sigma_{l}$ a standard sphere; otherwise $\Sigma_{l}$ has torsion in its homology of dimension $l-1$.

An interesting subclass of the equivariant metrics on $\Sigma_{l}$ comes from those equivariantly and isometrically imbedded in $C^{n+1}$ with the same orbit structure as our Brieskorn example above. We shall call these special. Then at the point 
$\left(x_{0}, y_{0}, x_{1}, y_{1}, x_{2}, y_{2}\right) \in \bar{M} \subset C^{3}$, the induced metric satisfies

$$
\begin{array}{ll}
a_{11}=x_{1}^{2}+y_{1}^{2}, & b_{11}=x_{1}^{2}+x_{2}^{2}+y_{1}^{2}+y_{2}^{2}, \\
a_{12}=x_{1} x_{2}+y_{1} y_{2}, & b_{12}=2 l\left(x_{2} y_{1}-x_{1} y_{2}\right), \\
a_{22}=x_{2}^{2}+y_{2}^{2}, & b_{22}=4\left(x_{0}^{2}+y_{0}^{2}\right)+l^{2}\left(x_{1}^{2}+x_{2}^{2}+y_{1}^{2}+y_{2}^{2}\right) .
\end{array}
$$

Note that $\Sigma$ special means there are only four independent functions $a_{i j}$ and $b_{i j}$ since $b_{11}=\operatorname{Tr} a$ and $b_{12}^{2}=4 l^{2} A$. We will see in $\S \mathrm{V}$ that this simplifies the curvature of $\Sigma$.

One might ask which curves in the orbit space of $C^{n+1}$ by $G$ correspond to smooth manifolds equivariantly diffeomorphic to $\Sigma_{l}$. To understand the behavior near singularities, note that for $G$ acting on $C^{n+1}$, we have isotropy group $H_{0}=S^{1}$ $\times O(n-2)$ only at points $(0, X, Y) \in R^{2} \times R^{n} \times R^{n}$ with $X \cdot X=Y \cdot Y \neq 0$ and $X \cdot Y=0$. The slice representation of $H_{0}$ is modelled on $S^{1}$ winding twice in one plane (corresponding to the part of the slice in $\Sigma_{l}$ ), $2 l$ times in another plane, and $S^{1}$ acting trivially on a third line. For $S^{1}$ acting on $C^{2} \times R$ by $\lambda\left(z_{1}, z_{2}, x_{3}\right)=$ $\left(\lambda^{2} z_{1}, \lambda^{2 l_{2}} z_{2}, x_{3}\right)$, the invariant functions are generated by

$$
A_{1}=\left|z_{1}\right|^{2}, \quad A_{2}=\left|z_{2}\right|^{2}, \quad A_{3}+i A_{4}=z_{1}^{l} \bar{z}_{2}, \quad A_{5}=x_{3} .
$$

For $\Sigma \subset C^{2} \times R$ to be smooth with $T_{(0,0)} \Sigma \subset C \times\{0\}, \Sigma$ must be expressible locally near zero as the graph of an equivariant map $q$ : $C \rightarrow C \times R$, i.e., $z_{2}=$ $z_{1}^{l} q_{1}\left(A_{1}\right)$ and $x_{3}=q_{2}\left(A_{1}\right)$ where $q$ is $C^{\infty}$. For the image curve of $\Sigma$ in the orbit space, this means $A_{2}=A_{1}^{l}\left|q_{1}\left(A_{1}\right)\right|^{2}, A_{3}+i A_{4}=A_{1}^{l} \overline{q_{1}}\left(A_{1}\right), A_{5}=q_{2}\left(A_{1}\right)$. By explicitly choosing a slice in $C^{n+1}$ and translating this result, we obtain

Proposition IV.2. In order to give a smooth manifold equivariantly diffeomorphic to $\Sigma^{\prime}$ near the orbit of type $S^{1} \times O(n-2)$, it is necessary that near zero the image curve in the orbit space of $C^{n+1}$ be of the form

$$
A_{2}=\left|\psi_{1}\left(A_{1}\right)\right|^{2} A_{1}^{l}, \quad A_{5}=\psi_{2}\left(A_{1}\right), \quad A_{3}+i A_{4}=\psi_{1}\left(A_{1}\right) A_{1}^{l},
$$

where $\psi=\left(\psi_{1}, \psi_{2}\right): R \rightarrow C \times R$ is $C^{\infty}$.

One can also ask rigidity questions such as when are two special manifolds $\Sigma_{l}$ equivariantly isometric? It is easy to see that such an isometry must preserve the invariant functions $A_{5}, A_{1}$, and $A_{2}$, but $A_{3}$ and $A_{4}$ are only constrained by $A_{3}^{2}+A_{4}^{2}=A_{1}^{l} A_{2}$. (This circle of indeterminacy corresponds to the natural $S^{1}$ action on the orbit space $C^{n+1} / G$ given by the centralizer of $G$ in $O(2 n+2)$.)

V. The homogeneous space $S^{1} \times S O(n) / S O(n-2)$. In this section, we will outline the explicit calculation of the curvature tensor of the homogeneous space $S^{1} \times S O(n) / S O(n-2)$ which occurs as principal orbit (up to coverings and components) for the $S^{1} \times O(n)$ action on $\Sigma_{l}$. The results are summarized in Theorems V.1 and V.2 below. 
THEOREM V.1. (A) The nonzero components of the Ricci tensor of an invariant metric on $S^{1} \times S O(n) / S O(n-2)$ are given by

(4) $\operatorname{Ric}\left(E_{i}, E_{i}\right)=(n-2)-b_{22} \operatorname{Tr} a / B+a_{11} b_{22}(\operatorname{Tr} a)^{2} /(2 A B)-a_{11} b_{11} /(2 A)$,

(5) $\operatorname{Ric}\left(F_{i}, F_{i}\right)=(n-2)-b_{22} \operatorname{Tr} a / B+a_{22} b_{22}(\operatorname{Tr} a)^{2} /(2 A B)-a_{22} b_{11} /(2 A)$,

$$
\operatorname{Ric}(Q, Q)=(n-2) b_{12}^{2} /(2 A),
$$

$$
\operatorname{Ric}(P, P)=(n-2)\left[2+\left(b_{11}^{2}-(\operatorname{Tr} a)^{2}\right) /(2 A)\right] \text {, }
$$

$$
\operatorname{Ric}(P, Q)=(n-2) b_{11} b_{12} /(2 A) \text {, }
$$

(B) The scalar curvature is given by

$$
\kappa=\frac{(n-2)}{A}\left[(n-2) \operatorname{Tr} a-\frac{b_{11}}{2}+\frac{b_{22}}{2 B}\left(4 A-(\operatorname{Tr} a)^{2}\right)\right] .
$$

Theorem V.2. Modulo the obvious symmetries and skew symmetries (including Bianchi identity), the nonzero components of the curvature tensor for $S^{1} \times$ $S O(n) / S O(n-2)$ are given by

$$
\begin{gathered}
\langle R(P, Q) Q, P\rangle=0, \\
\left\langle R\left(E_{i}, P\right) P, E_{i}\right\rangle=b_{11}-a_{22}+a_{11}\left(b_{11}-\operatorname{Tr} a\right)^{2} /(4 A) \\
\left\langle R\left(F_{i}, P\right) P, F_{i}\right\rangle=b_{11}-a_{11}+a_{22}\left(b_{11}-\operatorname{Tr} a\right)^{2} /(4 A), \\
\left\langle R\left(E_{i}, P\right) P, F_{i}\right\rangle=a_{12}+a_{12}\left(b_{11}-\operatorname{Tr} a\right)^{2} /(4 A), \\
\left\langle R\left(E_{i}, Q\right) Q, E_{i}\right\rangle=a_{11} b_{12}^{2} /(4 A), \\
\left\langle R\left(F_{i}, Q\right) Q, F_{i}\right\rangle=a_{22} b_{12}^{2} /(4 A), \\
\left\langle R\left(E_{i}, Q\right) Q, F_{i}\right\rangle=a_{12} b_{12}^{2} /(4 A), \\
\left\langle R\left(E_{i}, P\right) Q, E_{i}\right\rangle=b_{12}\left[-1+\left(a_{11}\left(a_{11}-b_{11}\right)+a_{12}^{2}\right) y A\right], \\
\left\langle R\left(F_{i}, P\right) Q, F_{i}\right\rangle=b_{12}\left[-1+\left(a_{22}\left(a_{22}-b_{11}\right)+a_{12}^{2}\right) / A\right] . \\
\left\langle R\left(E_{i}, P\right) Q, F_{i}\right\rangle=b_{12} a_{12}\left(b_{11}-\operatorname{Tr} a\right) /(4 A), \\
\left\langle R\left(E_{i}, Q\right) P, F_{i}\right\rangle=b_{12} a_{12}\left(b_{11}-\operatorname{Tr} a\right) /(4 A), \\
\left\langle R\left(E_{i}, E_{j}\right) E_{k}, E_{m}\right\rangle=\left(a_{12}^{2} b_{22} / B-a_{11}\right) \cdot\left(\delta_{i k} \delta_{j m}-\delta_{j k} \delta_{i m}\right), \\
\left\langle R\left(E_{i}, E_{j}\right) E_{k}, F_{m}\right\rangle=-a_{12}\left(B+b_{22}\left(a_{11}-a_{22}\right)\right) /(2 B) \cdot\left(\delta_{i k} \delta_{j m}-\delta_{j k} \delta_{i m}\right), \\
\left\langle R\left(E_{i}, E_{j}\right) F_{k}, F_{m}\right\rangle=\left(b_{11} / 4-\operatorname{Tr} a / 2+b_{22}\left(a_{11}-a_{22}\right)^{2} /(4 B)\right) \\
\cdot\left(\delta_{i k} \delta_{j m}-\delta_{j k} \delta_{i m}\right), \\
\left\langle R\left(E_{i}, F_{j}\right) F_{k}, E_{m}\right\rangle=a_{12}^{2} b_{22} / B \delta_{j k} \delta_{i m}-\left(b_{11}-\operatorname{Tr} a\right) / 2 \delta_{i j} \delta_{k m} \\
+\left(b_{22}\left(a_{11}-a_{22}\right)^{2}-b_{11} B\right) /(4 B) \delta_{i k} \delta_{j m},
\end{gathered}
$$




$$
\begin{gathered}
\left\langle R\left(F_{i}, F_{j}\right) F_{k}, E_{m}\right\rangle=-a_{12}\left(B+b_{22}\left(a_{22}-a_{11}\right)\right) /(2 B)\left(\delta_{i k} \delta_{j m}-\delta_{j k} \delta_{i m}\right), \\
\left\langle R\left(F_{i}, F_{j}\right) F_{k}, F_{m}\right\rangle=\left(a_{12}^{2} b_{22} / B-a_{22}\right)\left(\delta_{i k} \delta_{j m}-\delta_{j k} \delta_{i m}\right) .
\end{gathered}
$$

Of course most of the zero components in the curvature tensor are due to Ad $S O(n-2)$ invariance. (The curvature tensor gives an invariant map $\wedge^{2}\left(T_{x} m\right) \rightarrow$ $\wedge^{2}\left(T_{x} m\right)$ which is accordingly restricted by the Schur lemma.) Still the answers are remarkably simple, and they simplify considerably further under the assumptions $b_{11}=\operatorname{Tr} a$ and $b_{12}^{2}=4 l^{2} A$ which arise when $\Sigma_{l}$ is naturally embedded in $R^{2 n+2}$.

In calculating, it saves redundancy to note that there is an inner automorphism $\alpha$ of $G=S^{1} \times S O(n)$ interchanging $E_{i}$ and $F_{i}$ while leaving $S O(n-2)$ fixed. This allows geometrically defined functions of the $F_{i}$ to be written down immediately upon determining the corresponding functions of the $E_{i}$.

LEMmA V. 3. The automorphism $\alpha$ of $S^{1} \times S O(n) / S O(n-2)$ interchanges $E_{i}$ and $F_{i}$, sends $P$ to $-P$ and leaves $Q$ fixed. Accordingly it induces an isometry from $S^{1} \times S O(n) / S O(n-2)$ with invariant quadratic forms

$$
a=\left(\begin{array}{ll}
a_{11} & a_{12} \\
a_{12} & a_{22}
\end{array}\right) \text { and } b=\left(\begin{array}{ll}
b_{11} & b_{12} \\
b_{12} & b_{22}
\end{array}\right)
$$

to $S^{1} \times S O(n-2)$ with quadratic forms

$$
\left(\begin{array}{ll}
a_{22} & a_{12} \\
a_{12} & a_{11}
\end{array}\right) \text { and }\left(\begin{array}{rr}
b_{11} & -b_{12} \\
-b_{12} & b_{22}
\end{array}\right)
$$

respectively.

The first step in calculating $R(X, Y) Z$ is to determine the bilinear form $U(X, Y)$ describing the connection. (At $e, \nabla_{X^{*}} Y^{*}=-\frac{1}{2}[X, Y]^{*}+U(X, Y)^{*}$, where $[X, Y]$ is taken in g.) Table V.1 describes the Lie bracket structure of $g$. (These relations follow from the calculation $\left[0_{a}^{b}, 0_{c}^{d}\right]=-\delta_{a c} 0_{b}^{d}+\delta_{a d} 0_{b}^{c}+\delta_{b c} 0_{a}^{d}-\delta_{b d} 0_{a}^{c}$.)

In stating $U(X, Y)$ it is convenient to introduce new elements of $\not p$ orthogonal to some of our standard basis elements. We will use the notation

$E_{k}^{\prime}=\left(a_{22} E_{k}-a_{12} F_{k}\right) / A, \quad F_{K}^{\prime}=\left(-a_{12} E_{k}+a_{11} F_{k}\right) / A, \quad P^{\prime}=\left(b_{22} P-b_{12} Q\right) / B$.

Notice $\left\langle E_{k}^{\prime}, F_{k}\right\rangle=0$ and $\left\langle E_{k}^{\prime}, E_{k}^{\prime}\right\rangle=\left\langle F_{k}, F_{k}\right\rangle / A$ with similar identities for the others. Also, as the parameter $b_{11}-\operatorname{Tr} a$ appears frequently, we will set $\tau=b_{11}$ $\operatorname{Tr} a$. Table V.2 summarizes the Lie bracket structure of our additional elements. Table V.3 gives $\langle U(X, Y), Z\rangle=\frac{1}{2}\left(\left\langle[Z, X]_{\mu}, Y\right\rangle+\left\langle X,[Z, Y]_{\not k}\right\rangle\right)$. Using this, it is quite easy to verify

Proposition V.4. The nonzero components of $U(X, Y)$ are given by

$$
\begin{gathered}
U\left(E_{k}, E_{k}\right)=a_{12} P^{\prime}, \\
U\left(E_{k}, F_{k}\right)=\left(a_{22}-a_{11}\right) P^{\prime},
\end{gathered}
$$




$$
\begin{gathered}
U\left(F_{k}, F_{k}\right)=-a_{12} P^{\prime}, \\
U\left(E_{k}, Q\right)=b_{12} E_{k}^{\prime}, \\
U\left(F_{k}, Q\right)=-b_{12} F_{k}^{\prime}, \\
U\left(E_{k}, P\right)=-\left(F_{k}+\tau F_{k}^{\prime}\right) / 2, \\
U\left(F_{k}, P\right)=-\left(E_{k}+\tau E_{k}^{\prime}\right) / 2 .
\end{gathered}
$$

The curvature tensor $R(X, Y) Z$ is given by the sum of seven term $(\mathbf{K N})$ :

$R(1):-\left[[X, Y]_{h}, Z\right]$,

$R(2):-\left[[X, Y]_{k}, Z\right]_{k} / 2$.

$R(3):-U\left([X, \bar{Y}]_{\not h}, \bar{Z}\right)$,

$R(4):\left(\left[X,[Y, Z]_{\underline{\prime l}}\right]_{\underline{k}}-\left[Y,[X, Z]_{\underline{k}^{2}}\right]_{\underline{k}}\right) / 4$,

$R(5):\left(U\left(X,[Y, Z]_{p h}\right)-U\left(Y,[X, Z]_{p h}\right) / 2\right.$,

$\left.R(6):\left([U(X, Z), Y]_{p}-U(Y, Z), X\right]_{p}\right) / 2$,

$R(7): U(X, U(Y, Z))-U(Y, U(X, \bar{Z}))$.

In view of this, it is natural to prove the following:

LEMma V.5. The nonzero components of $[U(X, Y), Z]_{k h}$ and $U(U(X, Y), Z)$ are as given in Tables V.4 and V.5. (Note that the last column gives a common factor which should be multiplied by each entry in the given row.)

It is now fairly straightforward to calculate $R(X, Y) Z$ and simplify. As V.2 indicates, a remarkable amount of algebraic simplification occurs. (To calculate $\operatorname{Ric}(X, Y)$ in V.1, it is convenient to use the orthogonal basis $\left\{E_{i}, E_{i}^{\prime}, P, P^{\prime}\right\}$ of $\not$. For orthonormal bases $\left\{e_{i}\right\}, \operatorname{Ric}(u, v)=\sum_{i}\left\langle R\left(e_{i}, u\right) v, e_{i}\right\rangle$.)

The question of Einstein metrics on $S^{1} \times S O(n) / S O(n-2)$ is a natural one. If $\operatorname{Ric}(Q, Q)=c b_{22}$ and $\operatorname{Ric}(P, Q)=c b_{12}$ with $c \neq 0$, we see quickly that $b_{12} \neq 0$, $b_{11}=2 A c /(n-2)$, and $b_{22}=(n-2) b_{12}^{2} / 2 A c$. Hence $B=0$, contradicting the nondegeneracy of the metric. The Ricci flat case is handled by noting $b_{12}=0$ from $\operatorname{Ric}(Q, Q)$ and then

$$
\operatorname{Ric}\left(E_{i}, E_{i}\right)=\operatorname{Ric}\left(F_{i}, F_{i}\right)=0 \Rightarrow a_{11}=a_{22} \Rightarrow \operatorname{Ric}(P, P)=(n-2) b_{11}^{2} / 2 A \neq 0
$$

for nondegenerate metrics. So we have

THEOREM V.6. $S^{1} \times S O(n) / S O(n-2)$ does not admit a homogeneous Einstein metric.

(Theorem V.6 is well known as a consequence of the theorems of Bonnet-Myers and Bochner.)

When $b_{12}=0, S^{1} \times S O(n) / S O(n-2)$ splits isometrically, and thus our formulas give the Ricci tensor of the Stiefel manifold $S O(n) / S O(n-2)$ as well. If one starts by considering the equation $\operatorname{Ric}\left(E_{i}, E_{i}\right)-\operatorname{Ric}\left(F_{i}, F_{i}\right)=c\left(a_{11}-a_{22}\right)$, one 
fairly quickly finds that the only homogeneous Einstein metric on $S O(n) / S O(n-2)$ is proportional to the one given by $a_{11}=a_{22}=1, a_{12}=0$, and

$$
b_{11}=2(n-2) /(n-1) \text {. }
$$

This example was first discovered by Sagle [Sa].

THEOREM V.7. Any two homogeneous Einstein metrics on $S O(n) / S O(n-2)$ are homothetic.

TABLE V.1. $[X, Y]$

\begin{tabular}{|c|c|c|c|c|}
\multicolumn{1}{c}{$X$} & \multicolumn{1}{c}{$\left[X, E_{j}\right]$} & \multicolumn{1}{c}{$\left[X, F_{j}\right]$} & {$[X, P]$} & {$[X, Q]$} \\
\hline$E_{i}$ & $-0_{i}^{i}$ & $\delta_{i j} P$ & $-F_{i}$ & 0 \\
\hline$F_{i}$ & $-\delta_{i j} P$ & $-0_{i}^{j}$ & $E_{i}$ & 0 \\
\hline$P$ & $F_{j}$ & $-E_{j}$ & 0 & 0 \\
\hline$Q$ & 0 & 0 & 0 & 0 \\
\hline
\end{tabular}

$$
0_{i}^{i}=0
$$

TABLE V.2. $[X, Y]_{\underline{L}}$

\begin{tabular}{|c|c|c|c|}
\hline$X$ & $E_{k}$ & $F_{K}$ & $P$ \\
\hline$E_{h}^{\prime}$ & $\frac{a_{12} P}{A}$ & $\frac{a_{22} P}{A}$ & $\frac{-a_{22} F_{k}-a_{12} E_{k}}{A}$ \\
\hline$F_{h}^{\prime}$ & $\frac{-a_{11} P}{A}$ & $\frac{-a_{12} P}{A}$ & $\frac{a_{12} F_{k}+a_{11} E_{k}}{A}$ \\
\hline$P^{\prime}$ & $\frac{b_{22} F_{h}}{B}$ & $\frac{-b_{22} E_{k}}{B}$ & 0 \\
\hline
\end{tabular}

\begin{tabular}{|c|c|c|c|c|}
\hline$U(X, Y)$ & $\left\langle U(X, Y), E_{i}\right\rangle$ & $\left\langle U(X, Y), F_{i}\right\rangle$ & $\langle U(X, Y), P\rangle$ & $\langle U(X, Y), Q\rangle$ \\
\hline$U\left(E_{k}, E_{l}\right)$ & 0 & 0 & $\delta_{k \prime} a_{12}$ & 0 \\
\hline$U\left(E_{k}, F_{l}\right)$ & 0 & 0 & $\delta_{k l} \frac{\left(a_{22}-a_{11}\right)}{2}$ & 0 \\
\hline$U\left(F_{k}, F_{l}\right)$ & 0 & 0 & $-\delta_{k l} a_{12}$ & 0 \\
\hline$U\left(E_{k}, P\right)$ & $\frac{-\delta_{i k} a_{12}}{2}$ & $\frac{\delta_{i k}\left(a_{11}-b_{11}\right)}{2}$ & 0 & 0 \\
\hline$U\left(E_{k}, Q\right)$ & 0 & $\frac{-\delta_{i k} b_{12}}{2}$ & 0 & 0 \\
\hline$U\left(F_{k}, P\right)$ & $\delta_{i k} \frac{\left(b_{11}-a_{22}\right)}{2}$ & $\frac{\delta_{i k} a_{12}}{2}$ & 0 & 0 \\
\hline$U\left(F_{k}, Q\right)$ & $\delta_{i k} \frac{b_{12}}{2}$ & 0 & 0 & 0 \\
\hline $\begin{array}{l}U(P, P) \\
U(P, Q)\end{array}$ & 0 & 0 & 0 & 0 \\
\hline$U(Q, Q)$ & 0 & 0 & 0 & 0 \\
\hline
\end{tabular}

TABLE V.3. $\langle U(X, Y), Z\rangle$ 
TABLE V.4. Nonzero $[U(X, Y), Z]_{n}$

\begin{tabular}{|c|c|c|c|c|}
\hline$U(X, Y)$ & $E_{i}$ & $F_{i}$ & $P$ & $\begin{array}{c}\text { Common } \\
\text { Factor }\end{array}$ \\
\hline$U\left(E_{j}, E_{h}\right)$ & $F_{i}$ & $-E_{i}$ & 0 & $\delta_{j k} \frac{a_{12} b_{22}}{B}$ \\
\hline$U\left(E_{,}, F_{h}\right)$ & $F_{i}$ & $-E_{i}$ & 0 & $\delta_{j k} \frac{b_{22}}{2 B}\left(a_{22}-a_{11}\right)$ \\
\hline$U\left(F_{j}, F_{h}\right)$ & $F_{i}$ & $-E_{i}$ & 0 & $-\delta_{j k} \frac{a_{12} b_{22}}{B}$ \\
\hline$U\left(E_{j}, P\right)$ & $\delta_{i j}\left(A+\tau a_{11}\right) P$ & $\delta_{i j} \tau a_{12} P$ & $-A E_{j}-\tau\left(a_{11} E_{j}+a_{12} F_{j}\right)$ & $\frac{1}{2 A}$ \\
\hline$U\left(F_{j}, P\right)$ & $\delta_{i j} \tau a_{12} P$ & $\delta_{i j}\left(A+\tau a_{22}\right) P$ & $-A F_{j}-\tau\left(a_{22} F_{j}+a_{12} E_{j}\right)$ & $\frac{1}{2 A}$ \\
\hline$U\left(E_{j}, Q\right)$ & $\delta_{i j} a_{11} P$ & $\delta_{i j} a_{12} P$ & $-\left(a_{11}\left(E_{j}+a_{12} F_{j}\right)\right)$ & $\frac{b_{12}}{2 A}$ \\
\hline$U\left(F_{i}, Q\right)$ & $\delta_{i j} a_{12} P$ & $\delta_{i j} a_{22} P$ & $-\left(a_{12} E_{j}+a_{22} F_{j}\right)$ & $\frac{b_{12}}{2 A}$ \\
\hline
\end{tabular}

TABLE V.5. $U(U(X, Y), Z)$

\begin{tabular}{|c|c|c|c|c|c|}
\hline X.Y) & $E$ & $F_{i}$ & $r$ & $Q$ & $\begin{array}{l}\text { Common } \\
\text { Factor }\end{array}$ \\
\hline$\left(1 l_{,}, l_{i_{\alpha}}\right)$ & 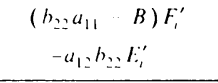 & $\begin{array}{l}a_{12} b_{22} F_{i}^{\prime} \\
\cdot\left(\begin{array}{ll}B & b_{22} a_{22}\end{array}\right) l_{i}^{\prime}\end{array}$ & () & () & $\frac{\delta_{t h} a_{12}}{2 B}$ \\
\hline$\left(\left(I_{i}, F_{h}\right)\right.$ & $\begin{array}{l}\text { same as } \\
\text { above }\end{array}$ & $\begin{array}{l}\text { same as } \\
\text { above }\end{array}$ & () & 0 & $\frac{\delta_{1 k}\left(a_{22} a_{11}\right)}{4 B}$ \\
\hline$\left(\left(F_{,}, F_{h}\right)\right.$ & $\begin{array}{l}\text { same as } \\
\text { above }\end{array}$ & $\begin{array}{c}\text { same as } \\
\text { above }\end{array}$ & () & () & $\frac{-\delta_{1 k} a_{12}}{2 B}$ \\
\hline$\left(\left(I_{i}, P\right)\right.$ & $\begin{array}{r}\delta_{t h} P^{\prime}\left[\tau\left(a_{11}^{2}+a_{12}^{2}\right)\right. \\
\left.\cdot A\left(2 a_{11} b_{11}\right)\right]\end{array}$ & $\begin{array}{l}\delta_{t h} a_{12} \\
\quad \cdot P^{\prime}[\tau \operatorname{Tr} a+2.4]\end{array}$ & $\left(b_{11} \tau \quad A\right) I_{k}$ & 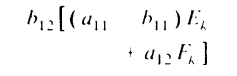 & $\frac{1}{4.4}$ \\
\hline$\left(\left(F_{k} \cdot P\right)\right.$ & $\begin{array}{l}\delta_{i k} a_{12} \\
\quad \cdot \operatorname{P}[\tau \operatorname{Tr} a+2 A]\end{array}$ & 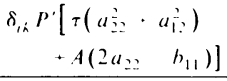 & $-\left(b_{11} \tau \quad A\right) F_{h}$ & $\begin{aligned} b_{12} & {\left[a_{12} E_{h}\right.} \\
& \left.+\left(a_{22}-b_{11}\right) F_{h}\right]\end{aligned}$ & $\frac{1}{4.4}$ \\
\hline$l\left(I_{L}, Q\right)$ & $\delta_{1, k} P^{\prime}\left[a_{11}^{2}+a_{12}^{2} \cdots A\right]$ & $\delta_{1 k} a_{12} \operatorname{Tr} a P^{\prime}$ & $\begin{array}{c}\left(a_{22}-b_{11}\right) l_{i h} \\
a_{12} F_{h}\end{array}$ & $-b_{12} l_{k}$ & $\frac{h_{12}}{4.4}$ \\
\hline$\ell\left(F_{h}, Q\right)$ & $\delta_{t h} a_{12} \operatorname{Tr} a P^{\prime}$ & $\delta_{t h} P^{\prime}\left\{a_{22}^{2} \cdot a_{12}^{\prime}, \quad A\right\}$ & $\begin{array}{c}\left(\begin{array}{ll}a_{11} & b_{11}\end{array}\right) F_{h} \\
a_{12} l_{h}\end{array}$ & $-h_{12} F_{h}$ & $\frac{h_{12}}{4.4}$ \\
\hline
\end{tabular}

VI. Curvature formulas for Kervaire spheres. Using the results of §III it is now very easy to determine the components in orbital directions of manifolds such as the $\Sigma_{l}$ described in $\S$ I. The remaining components are covered in the following theorem which follows by computation from III.2 and V.2.

THEOREM VI.1. Let $M$ be an almost homogeneous manifold with principal orbit type $S^{1} \times S O(n) / S O(n-2)$. Then (up to the usual curvature symmetries), the nonzero components involving $\hat{L}$ are given by

$$
\begin{aligned}
& \langle R(\hat{L}, P) P, \hat{L}\rangle=-\hat{L} \hat{L} b_{11} / 2+\left[\hat{L} b_{11} \hat{L} B-b_{11} \operatorname{det} \hat{L} b\right] / 4 B, \\
& \langle R(\hat{L}, P) Q, \hat{L}\rangle=-\hat{L} \hat{L} b_{12} / 2+\left[\hat{L} b_{12} \hat{L} B-b_{12} \operatorname{det} \hat{L} b\right] / 4 B,
\end{aligned}
$$


(4) $\left\langle R\left(\hat{L}, E_{i}\right) E_{i}, \hat{L}\right\rangle=-\hat{L} \hat{L} a_{11} / 2+\left[\hat{L} a_{11} \hat{L} A-a_{11} \operatorname{det} \hat{L} a\right] / 4 A$,

(5) $\left\langle R\left(\hat{L}, E_{i}\right) F_{i}, \hat{L}\right\rangle=-\hat{L} \hat{L} a_{12} / 2+\left[\hat{L} a_{12} \hat{L} A-a_{12} \operatorname{det} \hat{L} a\right] / 4 A$,

(6) $\left\langle R\left(\hat{L}, F_{i}\right) F_{i}, \hat{L}\right\rangle=-\hat{L} \hat{L} a_{22} / 2+\left[\hat{L} a_{22} \hat{L} A-a_{22} \operatorname{det} \hat{L} a\right] / 4 A$,

(9) $\left\langle R\left(E_{i}, P\right) E_{i}, L\right\rangle=\frac{1}{2}\left[-\alpha L a_{11}-\left(\beta+\frac{3}{2}\right) L a_{12}+a_{12}\left(b_{22} L b_{11}-b_{12} L b_{12}\right) / B\right]$,

(10) $\left\langle R\left(E_{i}, P\right) F_{i}, L\right\rangle=\frac{1}{2}\left[\left(\frac{1}{2}-\gamma\right) L a_{11}+\alpha L a_{12}+\left(L b_{11}-2 L a_{22}\right) / 2\right.$

$$
\left.+\left(a_{22}-a_{11}\right)\left(b_{22} L b_{11}-b_{12} L b_{12}\right) /(2 B)\right] \text {, }
$$

(11) $\left\langle R\left(F_{i}, P\right) E_{i}, L\right\rangle=\frac{1}{2}\left[-\alpha L a_{12}-\left(\beta+\frac{1}{2}\right) L a_{22}+\left(2 L a_{11}-L b_{11}\right) / 2\right.$

$$
\left.+\left(a_{22}-a_{11}\right)\left(b_{22} L b_{11}-b_{12} L b_{12}\right) /(2 B)\right],
$$

(12) $\left\langle R\left(F_{i}, P\right) F_{i}, L\right\rangle=\frac{1}{3}\left[\left(\frac{3}{2}-\gamma\right) L a_{12}+\alpha L a_{22}+a_{12}\left(b_{12} L b_{12}-b_{22} L b_{11}\right) / B\right]$,

(13) $\left\langle R\left(E_{i}, Q\right) E_{i}, L\right\rangle=\left[b_{12}\left(a_{11} L a_{12}-a_{12} L a_{11}\right) /(4 A)\right.$

$$
\left.+a_{12}\left(-b_{12} L b_{22}+b_{22} L b_{12}\right)\right] /(2 B),
$$

(14) $\left\langle R\left(F_{i}, Q\right) F_{i}, L\right\rangle=\left[b_{12}\left(a_{12} L a_{22}-a_{22} L a_{12}\right) /(4 A)\right.$

$$
\left.+a_{12}\left(b_{12} L b_{22}-b_{22} L b_{12}\right) /(2 B)\right] \text {, }
$$

(15) $\left\langle R\left(E_{i}, Q\right) F_{i}, L\right\rangle=b_{12}\left(a_{12} L a_{12}-a_{22} L a_{11}\right) /(4 A)$

$$
+L b_{12} / 4+\left(a_{11}-a_{22}\right)\left(b_{12} L b_{22}-b_{22} L b_{12}\right) /(4 B),
$$

(16) $\left\langle R\left(F_{i}, Q\right) E_{i}, L\right\rangle=b_{12}\left(a_{11} L a_{22}-a_{12} L a_{12}\right) /(4 A)$

$$
-L b_{12} / 4+\left(a_{11}-a_{22}\right)\left(b_{12} L b_{22}-b_{22} L b_{12}\right) /(4 B),
$$

where $\alpha=\tau a_{12} /(2 A), \beta=-\frac{1}{2}-a_{11} \tau /(2 A), \gamma=\frac{1}{2}+a_{22} \tau /(2 A)$, and $\tau=b_{11}-$ $a_{11}-a_{22}$.

It is now quite easy to determine the Ricci tensor of $M$. If we introduce the differential operators $T_{1}$ and $T_{2}$ below, the results are then easy to state.

$$
\begin{aligned}
& T_{1} f=\frac{-\hat{L} \hat{L} f}{2}-\frac{f \operatorname{det} \hat{L} a}{2 A}+\hat{L} f\left[-\frac{\hat{L} \log B}{4}+\frac{(4-n)}{4} \hat{L} \log A\right] \\
& T_{2} F=\frac{-\hat{L} \hat{L} f}{2}-\frac{f \operatorname{det} \hat{L} b}{2 B}+\hat{L} f\left[\frac{\hat{L} \log B}{4}+\frac{(2-n)}{4} \hat{L} \log A\right]
\end{aligned}
$$


THEOREM VI.2. Let $M$ be as in IV.1. The Ricci tensor of $M$ is given by (where $\widehat{\mathrm{Ric}}$ is in V.1)

$$
\begin{aligned}
& \operatorname{Ric}\left(E_{i}, E_{i}\right)=\widehat{\operatorname{Ric}}\left(E_{i}, E_{i}\right)+T_{1} a_{11}, \\
& \operatorname{Ric}\left(E_{i}, F_{i}\right)=\widehat{\operatorname{Ric}}\left(E_{i}, F_{i}\right)+T_{1} a_{12}, \\
& \operatorname{Ric}\left(F_{i}, F_{i}\right)=\widehat{\operatorname{Ric}}\left(F_{i}, F_{i}\right)+T_{1} a_{22}, \\
& \operatorname{Ric}(P, P)=\widehat{\operatorname{Ric}}(P, P)+T_{2} b_{11}, \\
& \operatorname{Ric}(P, Q)=\widehat{\operatorname{Ric}}(P, Q)+T_{2} b_{12}, \\
& \operatorname{Ric}(Q, Q)=\widehat{\operatorname{Ric}}(Q, Q)+T_{2} b_{22}, \\
& \operatorname{Ric}(\hat{L}, \hat{L})=\frac{-\hat{L} \hat{L} B}{2 B}+\frac{(\hat{L} \log B)^{2}}{4}+\frac{\operatorname{det} \hat{L} b}{2 B} \\
&+(n-2)\left[-\frac{\hat{L} \hat{L} A}{2 A}+\frac{(\hat{L} \log A)^{2}}{4}+\frac{\operatorname{det} \hat{L} a}{2 A}\right], \\
& \operatorname{Ric}(\hat{L}, P)=\frac{(n-2)}{2 A}\left[a_{12}\left(\hat{L} a_{11}-\hat{L} a_{22}\right)+\left(a_{22}-a_{11}\right) \hat{L} a_{12}\right], \\
& \operatorname{Ric}(\hat{L}, Q)=0 .
\end{aligned}
$$

Proof. (1) and (2) are immediate upon noting $\log C=\frac{1}{2}(\log B+(n-2) \log A)$ and using our observation about $(\hat{L} h) h^{-1}(\hat{L} h)$ for two-by-two matrices. (3) utilizes the sectional curvature formulae above.

We would like to make several remarks about curvature realizability questions. First, by virtue of invariant theory (Theorem IV.1), when $l>1, \hat{L} b=\hat{L} a=0$ at $r=0$. Thus the curvature tensor of $\Sigma$ restricted to this singular orbit is equal to the curvature tensor of a homogeneous metric on a Stiefel manifold. Since Stiefel manifolds $O(n) / O(n-2)$ do not have homogeneous metrics of positive curvature [Be and W], we obtain (without using VI.1)

THEOREM VI.3. When $l>1, \Sigma_{\text {l }}$ does not have an equivariant metric of positive curvature in any neighborhood of the orbit of type $S^{1} \times O(n-2)$.

Cheeger has shown that equivariant metrics of positive Ricci curvature are possible.

We also remark that it is not difficult to combine the results of §IV with the above formulas to obtain curvature near the singular orbits. For example, near $r=0$, $B=4 b_{0} r^{2}+O\left(r^{4}\right)$. Since $b$ is an even function of $r$, terms like $\operatorname{det}(\hat{L} b) / B$ and $\hat{L} b \hat{L} \log B$ have well-behaved limits at $r=0$.

One can study the Ricci-tensor or Einstein metric realizability questions near zero by power series. As questions of formal power series, there do not seem to be local obstructions beyond those given by invariant theory. The question of convergence has not yet been resolved.

We also note that the contracted Bianchi identity $\delta$ Ric $=\frac{1}{2} d \kappa$ is useful in understanding VI.2. For example, in looking for Einstein metrics, we find that if the Ricci tensor along the fibers is proportional to the metric and $\operatorname{Ric}(\hat{L}, P)=0$ (i.e. 
$a_{11}-a_{22}$ and $a_{12}$ are proportional), then

$$
\hat{L}(\operatorname{Ric}(\hat{L}, \hat{L}))=\hat{L}\left(\log B A^{n-2}\right)(1-\operatorname{Ric}(\hat{L}, \hat{L}))
$$

allowing one to establish that the metric is $\operatorname{Einstein} i f \operatorname{Ric}(\hat{L}, \hat{L})$ is initially 1 .

VII. Cheeger's example. In [C], Cheeger elegantly bypassed many of the computations of this paper and sketched the construction of metrics of positive Ricci curvature on the Kervaire sphere. His strategy produced invariant metrics of a restricted kind. In this section, we will explain how his approach fits in with ours.

An equivariant tubular neighborhood of an orbit of type $K$ is given by $G \times{ }_{K} S$ which is the quotient of $G \times S$ by a $K$-action. Hence the product of a left $G$-invariant, right $K$-invariant metric on $G$ with a left $K$-invariant metric on $S$ will induce, by submersion, a left $G$-invariant metric on the tubular neighborhood. This is the basic construction of $[\mathbf{C}]$.

At $r=0$, the isotropy group $H_{0}$ is $S^{1} \times O(n-2)$. The adjoint representation of $G$ restricted to $H_{0}$ is $\operatorname{Ad}_{S O(n-2)} \oplus \rho_{n-2} \otimes \rho_{1}^{l} \oplus 2 \theta$, so suitable metrics on $g$ (before submersion) will satisfy

$$
\begin{gathered}
\left(\begin{array}{cc}
\left\langle E_{i}, E_{i}\right\rangle & \left\langle E_{i}, F_{i}\right\rangle \\
\left\langle F_{i}, E_{i}\right\rangle & \left\langle F_{i}, F_{i}\right\rangle
\end{array}\right)=\left(\begin{array}{cc}
a_{0} & 0 \\
0 & a_{0}
\end{array}\right), \\
\left(\begin{array}{cc}
\langle P, P\rangle & \langle P, Q\rangle \\
\langle Q, P\rangle & \langle Q, Q\rangle
\end{array}\right)=\left(\begin{array}{ll}
s_{11} & s_{12} \\
s_{12} & s_{22}
\end{array}\right)
\end{gathered}
$$

for constants $a_{0}, s_{11}, s_{12}$, and $s_{22}$; the remaining Lie algebra of the $S O(n-2)$ block is perpendicular to the above and will not affect the submersed metric. $D^{2}$ is given a metric $d r^{2}+f_{0}^{2} d \theta^{2}$ in polar coordinates, where $f_{0}$ is a function solely of $r$.

After submersion, we are basically measuring the inner products of the components of $E_{i}, F_{i}, P$, and $Q$ perpendicular to the isotropy $S^{1}$ which acts nontrivially on $G$ and $D^{2}$. Consequently the submersed metric becomes (in the notation of §IV)

$$
a=\left(\begin{array}{cc}
a_{0} & 0 \\
0 & a_{0}
\end{array}\right), \quad b=\frac{\hat{S}}{q}\left(\begin{array}{cc}
1 & -l \\
-l & l^{2}
\end{array}\right)+\frac{4 f_{0}^{2}}{q}\left(\begin{array}{ll}
s_{11} & s_{12} \\
s_{12} & s_{22}
\end{array}\right)
$$

where $\hat{S}=s_{11} s_{22}-s_{12}^{2}$ and $q=l^{2} s_{11}+2 l s_{12}+s_{22}+4 f_{0}^{2}$.

Near the other singular orbit $\bar{r}=0(r=L)$, the isotropy group is $H_{1}=Z_{2} \times$ $O(n-1)$ and invariant metrics on $G$ will satisfy

$$
\begin{gathered}
\left(\begin{array}{cc}
\langle P, P\rangle & \langle P, Q\rangle \\
\langle Q, P\rangle & \langle Q, Q\rangle
\end{array}\right)=\left(\begin{array}{cc}
b_{1} & 0 \\
0 & b_{2}
\end{array}\right), \\
\left(\begin{array}{cc}
\left\langle E_{i}, E_{i}\right\rangle & \left\langle E_{i}, F_{i}\right\rangle \\
\left\langle F_{i}, E_{i}\right\rangle & \left\langle F_{i}, F_{i}\right\rangle
\end{array}\right)=\left(\begin{array}{cc}
b_{1} & 0 \\
0 & a_{2}
\end{array}\right) .
\end{gathered}
$$


Combining this with a metric $d \bar{r}^{2}+f_{1}^{2} d \Theta^{2}$ on $D^{n-1}$ (spherical coordinates where $d \Theta^{2}$ is the canonical metric on the sphere $S^{n-2}$ and $f_{1}$ is a function solely of $\bar{r}$ ), we obtain a submersed metric satisfying

$$
a=\left(\begin{array}{cc}
b_{1} & 0 \\
0 & h
\end{array}\right), \quad b=\left(\begin{array}{cc}
b_{1} & 0 \\
0 & b_{2}
\end{array}\right)
$$

where $h=f_{1}^{2} a_{2} /\left(a_{2}+f_{1}^{2}\right)$.

The metrics are glued together in a region where $f_{0}$ and $f_{1}$ are constant; let $\bar{f}_{0}$ and $\bar{f}_{1}$ denote these respective constants and $\bar{q}=l^{2} s_{11}+2 l s_{12}+s_{22}+4 \bar{f}_{0}^{2}$. Requiring the two sets of equations for $a$ and $b$ to match up gives five equations in the nine variables $a_{0}, s_{11}, s_{12}, s_{22}, \bar{f}_{0}, b_{1}, b_{2}, a_{2}$, and $\bar{f}_{1}$. Using the equation for $b_{12}$, we obtain $\hat{S}=4 \bar{f}_{0}^{2} / l$. Set $\sigma=4 \bar{f}_{0}^{2} / \bar{q}, u=l s_{11}+s_{12}$, and $v=l s_{12}+s_{22}$. Plugging our expression for $\hat{S}$ into the equations for $b_{11}$ and $b_{22}$ gives $u=l b_{1} / \sigma, v=b_{2} / \sigma$. Now the three equations

$$
\hat{S}=\sigma \bar{q} s_{12} / l, \quad l u+v=(1-\sigma) \bar{q}, \quad \hat{S}=\left(u v-(l u+v) s_{12}\right) / l
$$

may be combined with those for $u$ and $v$ to solve first for $s_{12}$ and then for $s_{11}$ and $s_{22}$. Thus $a_{2}, b_{1}, b_{2}$ and $\sigma$ may be viewed as arbitrary parameters $(0<\sigma<1,0<$ $b_{1}<a_{2}$, and $\left.b_{2}>0\right)$ and the class of metrics obtained by Cheeger's construction may be described as follows:

(I) Region where the metric is induced from the tubular neighborhood about the Stiefel manifold $(r=0)$ :

$$
a=\left(\begin{array}{cc}
b_{1} & 0 \\
0 & b_{1}
\end{array}\right), \quad b=\left(\begin{array}{cc}
b_{1} & 0 \\
0 & b_{2}
\end{array}\right)+g\left(\begin{array}{cc}
l^{2} b_{1}^{2} & l b_{1} b_{2} \\
l b_{1} b_{2} & b_{2}^{2}
\end{array}\right)
$$

where

$$
g=\frac{1-\sigma}{\sigma \kappa}-\frac{1}{\sigma \kappa+4 f_{0}^{2} \sigma^{2}}
$$

Here $\kappa=l^{2} b_{1}+b_{2}$ and both $g$ and $f_{0}$ are functions of $r$. The value $\bar{f}_{0}$ of $f_{0}$ in the overlap region is given by $4 \bar{f}_{0}^{2}=\kappa /(1-\sigma)$. Also $f_{0}(0)=0, f_{0}^{\prime}(0)=1, g(0)=-1 / \kappa$, $g^{\prime \prime}(0)=8 / \kappa^{2}$, and the overlap value of $g$ is 0 .

(II) Region where the metric is induced from the tubular neighborhood about $S^{1} \times S^{n-1}(\bar{r}=0):$

$$
a=\left(\begin{array}{cc}
b_{1} & 0 \\
0 & h
\end{array}\right), \quad b=\left(\begin{array}{cc}
b_{1} & 0 \\
0 & b_{2}
\end{array}\right)
$$

where $h=\left(f_{1}^{2}-2\right) /\left(f_{1}^{2}+a_{2}\right)$ and $f_{1}$ are functions of $\bar{r}$ and the overlap value of $f_{1}$ is given by $\bar{f}_{1}^{2}=b_{1} a_{2} /\left(a_{2}-b_{1}\right)$. Additionally $f_{1}(0)=0, f_{0}^{\prime}(0)=1, h(0)=0$, $h^{\prime}(0)=0, h^{\prime \prime}(0)=2$, and $h=b_{1}$ in the overlap. 
Thus metrics of the kind utilized in $[\mathbf{C}]$ are specified by the length $L$ of the orbit space, four constants $a_{2}, b_{1}, b_{2}$, and $\sigma$, and the two functions $f_{0}$ and $f_{1}$ defined on complementary portions of the orbit space. General invariant metrics are specified by six functions $\left\{a_{i j}, b_{i j}\right\}$ and the length of the orbit space (Theorem IV.1).

In the region where description (I) is valid,

$$
\begin{aligned}
& \operatorname{Ric}(Q, Q)=\frac{(n-2) l^{2} b_{1}^{2} b_{2}^{2} g^{2}}{2 a_{1}^{2}}-\frac{b_{2}^{2} \ddot{g}}{2}+\frac{\kappa^{2} b_{22}^{2}}{r(1+k g)}, \\
& \operatorname{Ric}(P, P)=\frac{(n-2) b_{1}^{2}\left(1+l^{2} b_{1} g\right)^{2}}{2 a_{1}^{2}}-\frac{l^{2} b_{1}^{2} \ddot{g}}{2}+\frac{\kappa \dot{g}^{2} l^{2} b_{1}^{2}}{4(1+k g)}, \\
& \operatorname{Ric}(P, Q)=\frac{(n-2) l b_{1}^{2} b_{2} g\left(1+l^{2} b_{1} g\right)}{2 a_{1}^{2}}-\frac{l b_{1} b_{2} \ddot{g}}{2}+\frac{\kappa \dot{g}^{2} l b_{1} b_{2}}{4(1+\kappa g)}, \\
& \operatorname{Ric}\left(E_{i}, E_{i}\right)=(n-2)-\frac{1}{2}\left(1+l^{2} b_{11} g\right), \\
& \operatorname{Ric}\left(F_{i}, F_{i}\right)=\operatorname{Ric}\left(E_{i}, E_{i}\right), \\
& \operatorname{Ric}\left(E_{i}, F_{i}\right)=\operatorname{Ric}(P, \hat{L})=\operatorname{Ric}(Q, \hat{L})=0, \\
& \operatorname{Ric}(\hat{L}, \hat{L})=-\frac{1}{(1+\kappa g)^{1 / 2}} \hat{L} \hat{L}(1+\kappa g)^{1 / 2} .
\end{aligned}
$$

Here $\dot{g}=\hat{L} g=d g / d r$ and $\ddot{g}=\hat{L} \hat{L} g$.

A straightforward calculation gives

$$
\frac{\left[\operatorname{Ric}(P, P) \operatorname{Ric}(Q, Q)-\operatorname{Ric}(P, Q)^{2}\right]}{b_{1}^{2} b_{2}^{2}}=-\frac{(n-2)(1+\kappa g)^{1 / 2}}{2 \kappa a_{1}^{2}} \hat{L} \hat{L}(1+\kappa g)^{1 / 2}
$$

and

$$
\begin{aligned}
\frac{\operatorname{Ric}(P, P)}{b_{1}}+\frac{\operatorname{Ric}(Q, Q)}{b_{2}}= & \frac{(n-2)}{2 a_{1}^{2}}\left[b_{1}\left(1+l^{2} b_{1} g\right)^{2}+l^{2} b_{1}^{2} b_{2} g^{2}\right] \\
& -(1+\kappa g)^{1 / 2} \hat{L} \hat{L}(1+\kappa g)^{1 / 2} .
\end{aligned}
$$

We obtain the corollary:

Corollary VII.1. If $\hat{L} \hat{L}(1+\kappa g)^{1 / 2}<0$ and $-1 / \kappa<g<0$, then for $n \geqslant 3$, metrics of the form (I) give positive Ricci curvature on the neighborhood of the Stiefel manifold.

Since $\ddot{f}_{0}<0$ implies $\hat{L} \hat{L}(1+\kappa g)^{1 / 2}<0$, this corollary refines the positivity argument used by Cheeger in this region. 
Within the region where the metric is given by (II), the Ricci tensor is given by:

$$
\begin{aligned}
& \operatorname{Ric}(P, P)=(n-2)\left(1-\frac{h}{2 b_{1}}\right), \\
& \operatorname{Ric}(P, Q)=\operatorname{Ric}(Q, Q)=0, \\
& \operatorname{Ric}\left(E_{i}, E_{i}\right)=(n-2)-\frac{h}{2 b_{11}}, \\
& \operatorname{Ric}\left(F_{i}, F_{i}\right)=(n-3)+\frac{h^{2}}{2 b_{1}^{2}}-\frac{\ddot{h}}{2}+\frac{(4-n) \dot{h}^{2}}{4 h} \\
& =(n-3)\left(1-(\hat{L}(\sqrt{h}))^{2}\right)+\frac{h^{2}}{2 b_{1}^{2}}-\sqrt{h} \hat{L} \hat{L}(\sqrt{h}), \\
& \operatorname{Ric}\left(E_{i}, F_{i}\right)=\operatorname{Ric}(P, \hat{L})=\operatorname{Ric}(Q, \hat{L})=0, \\
& \operatorname{Ric}(\hat{L}, \hat{L})=(n-2)\left(-\frac{\ddot{h}}{2 h}+\frac{\dot{h}^{2}}{4 h^{2}}\right)=-\frac{(n-2)}{\sqrt{h}} \hat{L} \hat{L}(\sqrt{h}) .
\end{aligned}
$$

At $\bar{r}=0, h=0, \hat{L}(\sqrt{h})=1$, and $\hat{L} \hat{L}(\sqrt{h})=0$ in the limit. (We view $\hat{L}=\partial / \partial \bar{r}$.) Thus $\hat{L} \hat{L}(\sqrt{h}) \leqslant 0$ and $\hat{L}(\sqrt{h}) \geqslant 0$ implies $0 \leqslant \hat{L}(\sqrt{h}) \leqslant 1$. We obtain

COROLlaRY VII.2. If $\hat{L} \hat{L}(\sqrt{h})<0$ and $\sqrt{h}$ increases (as $\bar{r}$ increases) from 0 to $b_{1}$, then for $n \geqslant 3$, metrics of the form (II) in the neighborhood of the $S^{1} \times S^{n-1}$ orbit have nonnegative Ricci curvature.

Again, $\ddot{f}_{1}<0$ and $f_{1}$ increasing with the given boundary conditions implies the applicability of the above corollary and we are generalizing Cheeger's condition.

As in $[\mathbf{C}]$, these arguments suffice for obtaining nonnegative Ricci curvature on $\Sigma_{\text {. }}$. The obstructions to positivity lie in the fact that $\operatorname{Ric}(Q, Q)=0$ in region (II) and $\operatorname{Ric}(\hat{L}, \hat{L})=0$ along the $S^{1} \times S^{n-1}$ orbit and in the overlap ( $h=$ constant region). Deformations of the equivariant metric which solely involve $b_{22}$ and are supported away from the Stiefel manifold orbit are sufficient to realize positive Ricci curvature, as is to be expected from the result of Ehrlich cited in [C].

\section{REFERENCES}

[BdCH] A. Back, M. do Carmo, and W. Y. Hsiang, On some fundamental equations of equivariant riemannian geometry, preprint.

[Be] L. Bergery, Les variétés reimanniennes homogènes simplement connexes de dimension impaire a courbure strictement positive, J. Math. Pures Appl. 55 (1976), 47-68.

[Bi] E. Bierstone, General position of equivariant maps, Trans. Amer. Math. Soc. 234 (1977), 447-466.

[C] J. Cheeger, Some examples of manifolds of nonnegative curvature, J. Differential Geometry 8 (1973).

[HH] W. T. Hsiang and W. Y. Hsiang, On the construction of exotic and/or knotted minimal spheres in the standard Riemannian sphere by means of equivariant differential geometry, preprint.

[HL] W. Y. Hsiang and H. B. Lawson, Minimal submanifolds of low cohomogeneity, J. Differential Geometry 5 (1971), 1-38.

[KN] S. Kobayashi and K. Nomizu, Foundations of differential geometry. I, II, Interscience, New York, $1963,1969$. 
[L] D. Luna, Fonctions différentiables invariantes sous l'opération d'un groupe réductif, Ann. Inst. Fourier (Grenoble) 26 (1976), 33-49.

[ON] B. O'Neill, The fundamental equations of a submersion, Michigan Math. J. 13 (1966), 459-469.

[Sa] A. Sagle, Some homogeneous Einstein manifolds, Nagoya Math. J. 39 (1970), 81-106.

[Sc] G. Schwarz, Smooth functions invariant under the action of a compact Lie groups, Topology 14 (1975), 63-68.

[W] N. Wallach, Compact homogeneous riemannian manifolds with strictly positive curvature, Ann. of Math. 96 (1972), 277-295.

Department of Mathematical Sciences, State University of New York at Binghamton, BinghamTON, NeW YORK 13901

Department of Mathematics, University of California, Berkeley, California 94720 